\title{
El acento en el quechua de Chachapoyas: un sustrato de la lengua chacha o un vestigio protoquechua
}

\author{
Stress in Chachapoyas Quechua: a Linguistic Stratum of the Chacha Language \\ or a Proto-Quechua Vestige
}

\section{Jairo Valqui}

Universidad Nacional Mayor de San Marcos, Lima, Perú

Contacto: jvalquic@unmsm.edu.pe

https://orcid.org/0000-0003-1992-9795

\begin{abstract}
Resumen
Este artículo presenta datos preliminares del proyecto de investigación sobre los Patrones acentuales en el quechua de Chachapoyas y su implicancia para la reconstrucción del protoquechua, proyecto de tesis para el doctorado en Lingüística en la Universidad Nacional Mayor de San Marcos. El estado de la cuestión en torno al acento en el protoquechua no ha ofrecido hasta el momento una propuesta sobre cómo habrían sido sus patrones acentuales (cfr. Torero, 1964; Parker, 2013; Cerrón-Palomino, 2003). La hipótesis de nuestro proyecto es que los patrones acentuales del quechua de Chachapoyas conservan este aspecto prosódico del protoquechua y no de la lengua chacha estudiada por Taylor (2000) y Valqui y Ziemendorff (2016). En este artículo se presentan datos inéditos de característica acústica de esta variedad recolectados en pueblos de las provincias de Chachapoyas y Luya en el departamento de Amazonas. Además, se discuten estos datos, principalmente, con los patrones acentuales del quechua de Ferreñafe descritos por Escribens (1977) y los del quechua de Huancapón analizados por Pineda (1994).
\end{abstract}

Palabras clave: Quechua de Chachapoyas; Protoquechua; Quechua; Patrones acentuales.

\begin{abstract}
This article presents preliminary data of the research project on the Stress Patterns found in Chachapoyas Quechua and its Implications for the Reconstruction of Protoquechua. This is the thesis project for a PhD degree in Linguistics at the National University of San Marcos. The state of the art around the accent in protoquechua has not offered so far a proposal on how their accent patterns would have been like (Cf. Torero, 1964, Parker, 2013, Cerrón-Palomino, 2003). The hypothesis of our project is that the accentual patterns of Chachapoyas Quechua maintains its prosodic aspect from the protoquechua and not from the Chacha language studied by Taylor (2000) and Valqui \& Zie-
\end{abstract}


mendorff (2016). In this article, unpublished data of acoustic characteristics of this variety that were collected in towns of the provinces of Chachapoyas and Luya in the department of Amazonas are presented. In addition, these data are discussed, mainly, with the accentual patterns of Ferreñafe Quechua described by Escribens (1977) and those of Huancapón Quechua described by Pineda (1994).

Keywords: Strees Patterns; Chachapoyas Quechua; Protoquechua; Quechua.

Recibido: 01.12.17

Aceptado: 01.10.18

\section{Introducción}

Con la denominación quechua de Chachapoyas o quechua amazonense se hace referencia a una variedad quechua que se mantiene en algunos distritos de las provincias de Luya y Chachapoyas en el departamento de Amazonas. Agrupada con otras variedades como el de Lamas (San Martín), Ecuador y Colombia dentro del Quechua II B - Chínchay norteños para Torero (2007), Chínchay Septentrional para Cerrón-Palomino (2003)—, esta variedad, entre otras características, exhibe un marcado acento en la primera sílaba de las palabras que, según comentaba Parker (2013), ocasionaba la elisión de vocales y le configuraba de una fonética que lo distanciaba de las demás variedades quechuas.

Otro cambio en Amazonas ha sido el de acentuación a la primera sílaba de cada palabra y la subsecuente pérdida de vocales de ciertas sílabas no iniciales (se necesitaría un corpus más extenso para poder establecer $\mathrm{la}[\mathrm{s}]$ regla[s] exacta[s]). Los grupos consonánticos resultantes de hasta cuatro [consonantes] intervocálicas y tres en final de palabra le dan a este dialecto un sonido sorprendentemente noquechua. (Parker, 2013, p. 149)

Taylor (2000), uno de los investigadores con más estudios en esta variedad, señalaba que la fuerte acentuación de la primera sílaba en el quechua de Chachapoyas era una de las principales distinciones con la variedad lamista (San Martín), una de las variedades quechuas más cercanas en términos glotocronológicos (cfr. Torero, 1975, p. 241). Sobre este punto, Taylor pensaba que la evolución fonética del dialecto quechua de Amazonas se pudo deber a 
algún sustrato de la antigua lengua de los chachapuyas (cfr. Taylor, 2000, pp. 37-38). Sin embargo, el estado de la cuestión sobre el acento en otras variedades quechuas permite reconsiderar esta característica acentual como un vestigio de origen protoquechua. A favor de esta hipótesis se presentan en este artículo datos del quechua de Ferreñafe, ubicado en la subrama Quechua II, datos del quechua de Huancapón (Cajatambo), en la subrama del Quechua I, y datos inéditos de índole acústica del quechua de Chachapoyas ${ }^{1}$.

\section{El acento en el quechua de Ferreñafe y en el quechua de Huancapón}

En el quechua de Ferreñafe (Quechua II), de acuerdo con Escribens (1977), el acento presenta dos patrones: uno, donde recae en la penúltima sílaba de un lexema como en ['ma.may.ki.ta] /mama+yki+ta/ 'a tu madre'; y dos, donde recae en la penúltima sílaba de la palabra como sucede en [Ja. 'muy.gi] / $a \mathrm{amu}+\mathrm{nki} /$ 'vienes'. Aunque también encuentra casos de alternancia acentual como en ['ma.may.ki.ta] $\sim$ [ma.may. 'ki.ta], este autor se reafirma en los patrones aludidos anteriormente.

Hasta el momento parecería que nos hallamos ante dos pautas de acentuación: i) en la penúltima sílaba del lexema, ii) en la penúltima sílaba de la palabra. Cabe destacar que (ii) corresponde al patrón exclusivo de acentuación de la mayoría de variedades del quechua II, mientras que (i) se aproxima, en su rendimiento general, a la pauta bastante generalizada en quechua I, que acentúa la primera sílaba de la palabra (recuérdese que la mayoría de lexemas del quechua son disilábicos). (Escribens, 1977, p. 50)

De acuerdo con el autor, la elección de uno de estos patrones puede estar relacionada con la estructura sintáctica. Así, por ejemplo, en la frase [['mikunata] FN tşa'tfimun], el acento en la primera sílaba se aplica a la FN y el acento penúltimo al núcleo de la frase verbal. Para Escribens, la variedad quechua de Ferreñafe podría conservar el patrón acentual del protoquechua, “con alternancias entre las distribuciones enunciadas, que ha sido resuelta de maneras diferentes y más uniformes por los otros dialectos contemporáneos" (Escribens, 1977, p. 50).

En el quechua de Huancapón (Quechua I), de acuerdo con Pineda (1994), el acento de palabras constituidas por dos o tres sílabas mayoritariamente recae en la primera con la presencia de un acento secundario en las palabras trisilábicas 
como aparece en los nombres [á.Xa.lìn] 'tripas' y [sár.si.yò:] 'arete' o en los verbos [yá.na.Gày] 'acompañar' o [kú.su.rì:] 'cortar con cuchillo'. Así también, si se adicionan sufijos a lexemas constituidos por una o dos sílabas, el acento principal se mantiene en la primera y el acento secundario puede aparecer en el último o penúltimo lugar, como ocurre en [nóGatà] 'yo + acusativo', [páy.kù.na] $\sim$ [páy.kùn] 'ellos' o [páy.ku.ná.ta] 'ellos+acusativo'. Esta descripción le permite confirmar a la autora la presencia de una acentuación predominantemente sobre la primera sílaba de la palabra que caracteriza a esta variedad como una de las más conservadoras entre las variedades de la familia lingüística quechua.

Una pregunta que se origina de estas dos observaciones es si estos patrones acentuales de estas dos variedades quechuas de ramas distintas podrían estar relacionadas. Quizás, como sugiere Escribens (1977) para el quechua de Ferreñafe, los datos sobre el acento en el quechua de Huancapón (Pineda, 1994) y los datos que se presentarán en el siguiente acápite para el quechua de Chachapoyas nos pueden dar luces acerca de un patrón acentual más antiguo, posiblemente, como un rasgo prosódico heredado del protoquechua.

En Los dialectos quechuas, Torero(1964) estimaba que "las características prosódicas del protoquechua eran aproximadamente las de los dialectos modernos del departamento de Ancash y de las provincias de Cajatambo y Chancay, departamento de Lima" (Torero, 1964, p. 461). En adición a esta observación, Cerrón-Palomino (2003) agrega que los patrones acentuales de las variedades quechuas de Ferreñafe y Chachapoyas podrían ser también reminiscencias de un patrón acentual atribuible al protoquechua, como también parece evidenciar la variedad quechua que describió fray Domingo de Santo Tomás en 1560.

Hay, sin embargo, algunas variedades centro-norteño peruanas que poseen un régimen acentual diferente, en virtud del cual el rasgo culminativo es asignado a la primera sílaba con un acento secundario que recae en la penúltima, cuando la palabra contiene más de tres sílabas. Tales dialectos, entre los que caben mencionar a las hablas de Cajatambo y Chancay (Lima), Ancash, Ferreñafe (Lambayeque) y Amazonas, tal vez reflejen, como se dijo (cf. Torero 1964: 461-462), el patrón acentual originario atribuible al PQ. Ya se notó que dicho régimen acentual es reminiscencia del patrón culminativo descrito por Fray Domingo de Santo Tomás. (Cerrón-Palomino, 2003, p. 259) 
En efecto, los patrones acentuales que describe fray Domingo de Santo Tomás en su gramática quechua de 1560 presentan algunos rasgos prosódicos similares con las descripciones acentuales presentadas arriba para Ferreñafe y Huancapón, y ahora evidenciadas para Chachapoyas. Uno de esos patrones muestra que el acento puede recaer en la primera sílaba de una palabra trisilábica en nombres o verbos como ocurre en <máquiguan > 'con manos', <ñócanchic> 'nosotros' o <mícuni> 'yo como'; otro de los patrones muestra un acento en la penúltima sílaba o donde se encontraba una sílaba pesada.

Sacãse della regla todas las dictiones de dos syllabas, quando se les añade alguna particula por ornato, o por otra qualquier via, o la perposició, o articulo casual, que entõces, aunque tengã tres syllabas o mas guardan el mismo accento, que quando eran de dos syballas .v.g. Exemplo de lo primero (guauquilla) hermano (mácholla) viejo. Exẽplo de lo segundo (cingayoc) hõbre cõ narices (çabranac) hõbre del barbado Exẽplo de lo tercero (máquiguan) cõ manos (ñócaca) a mi Todos los cuales, ahquelos del primer exemplo con la particula (lla) puesta por ornato, y los del segundo, cõ las partículas (yoc) (nac) puestas por necessidad de la significaciõ, y los del tercero añadidas como preposiciones se hagã de tres syllabas, como consta, pero tienen el accento en la primera syllaba, como antes que se les añadiesen las dichas partículas. [...] Item, se sacan della regla, todos los términos de tres syllabas en que a la vocal de la primera syllaba, se siguen dos consonantes inmediatas, que las tales tienen el accẽto, en la primera sylaba v.g. (cóngori) rodillas (tóngori) nuez de la garganta (guachaquene) mujer que pare, y otras semejantes. (Santo Tomás, 1951, pp. 176-177)

\section{Datos preliminares sobre el acento en el quechua de Chachapoyas}

Los datos acústicos sobre la duración vocálica y el contorno tonal en palabras quechuas de la variedad chachapoyana evidencian que el acento principal recae generalmente en la primera sílaba de una palabra constituida por 3 o más sílabas. Las vocales que aparecen en la primera sílaba en palabras bisilábicas y trisilábicas mantienen un promedio de duración de $108 \mathrm{~ms}$, mientras aquellas que surgen en la segunda presentan un promedio de $82 \mathrm{~ms}$; es decir, que las vocales en el primer contexto duran más que las segundas, como se observa en el cuadro $1^{2}$. 
Cuadro 1. Promedio de vocales en la primera y segunda sílaba

\begin{tabular}{|c|c|c|}
\hline Vocales & Primera sílaba & Segunda sílaba \\
\hline$[\mathrm{i}]$ & 111 & 91 \\
\hline$[\mathrm{u}]$ & 102 & 81 \\
\hline$[\mathrm{a}]$ & 112 & 87 \\
\hline promedio & 108 & 86 \\
\hline
\end{tabular}

Fuente: Elaboración propia.

En la segunda sílaba o en la tercera, se reporta un alto porcentaje de vocales que tienden a acortarse, ensordecerse o incluso a elidirse por completo, como se observará en las figuras que presentan diversos sufijos. Por ejemplo, en la figura 1 se muestra el debilitamiento de la vocal dorsal alta /u/ de la palabra / pifku/ 'pájaro'. En este ejemplo, la vocal /u/ dura $53 \mathrm{~ms}$, es decir, $33 \mathrm{~ms}$ menos que el promedio presentado en el cuadro 1.

Figura 1. Debilitamiento de la alta dorsal en /pifku/ 'pájaro'

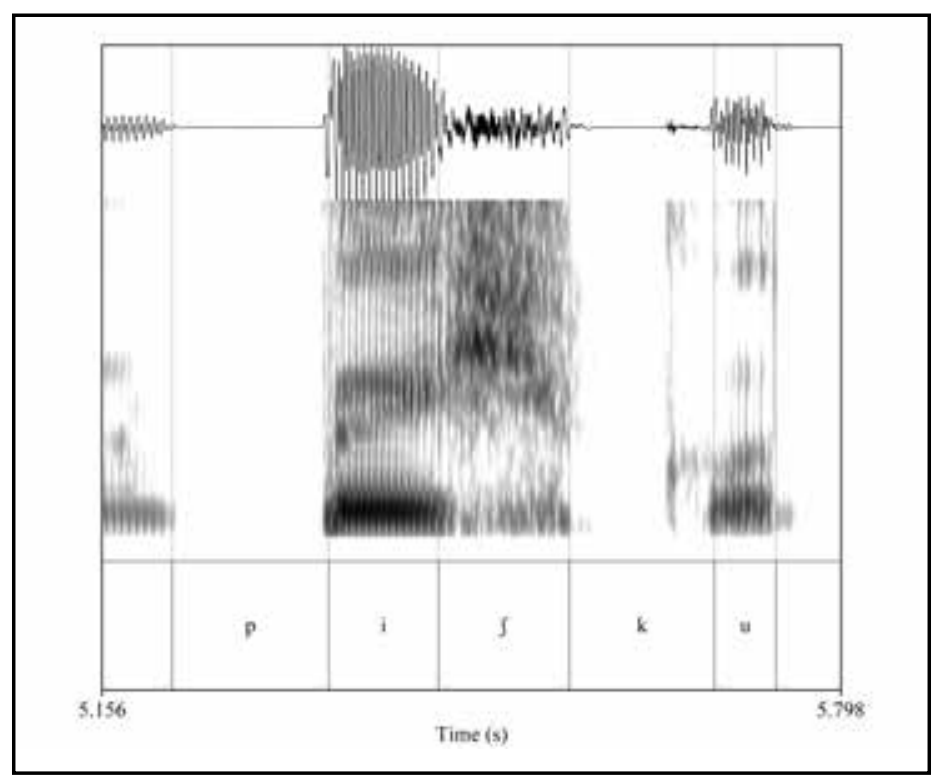

Fuente: Elaboración propia. 
La medición del contorno tonal, por ejemplo, en una palabra de tres sílabas como [si.ki.kÆ] </siki+2.a prs. pos./, como se puede apreciar en la figura 2, muestra el pico mayor en la vocal de la primera sílaba en relación con la segunda. El contorno tonal representado con líneas punteadas encima de las vocales delimitadas por líneas verticales no se presenta en la tercera vocal porque esta se encuentra completamente elidida.

Figura 2. Contorno tonal de la palabra /siki+2.a prs. pos./ 'tu trasero'

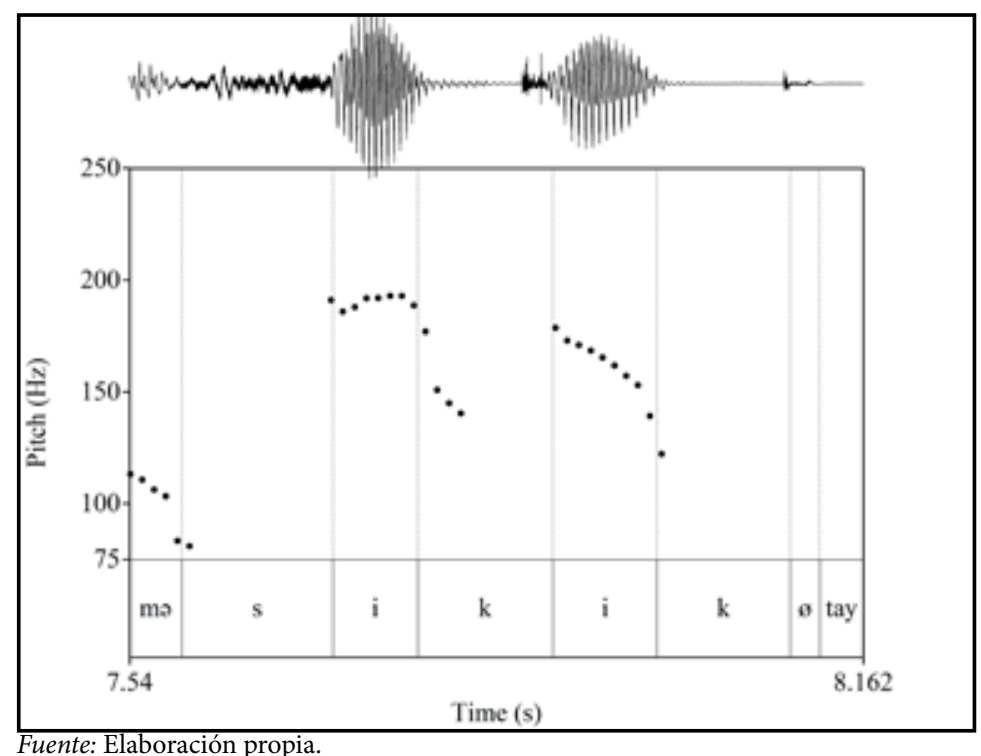

Fuente: Elaboración propia.

En una ventana de $20 \mathrm{~ms}$, el contorno tonal de la vocal [i] de la primera sílaba de la izquierda mide $196 \mathrm{~Hz}$ mientras que la otra vocal alta de la segunda sílaba mide $166 \mathrm{~Hz}$. Es decir, que la frecuencia del contorno tonal de la primera vocal es de 3 semitonos más alta que la segunda, y esta relación hace que se perciba la primera como más alta tonalmente que la segunda (para el análisis acústico del shipibo véase Elías-Ulloa, 2011). 
Si añadimos a la palabra /siki/ más sufijos que los posesivos como el locativo /-pi/ para formar una palabra de cuatro sílabas, veremos que el contorno tonal más alto se sigue manteniendo en la primera sílaba ['si.ki.kÆ.pæ] 'en tu trasero' como se muestra en la figura 3. Además, se puede observar la elisión de las vocales altas que formarían parte de los sufijos de segunda persona poseedora y del locativo.

Figura 3. Contorno tonal de palabra [.'si.ki.kØ.pØ.] </siki+2.a prs. pos.+loc./ 'en tu trasero'

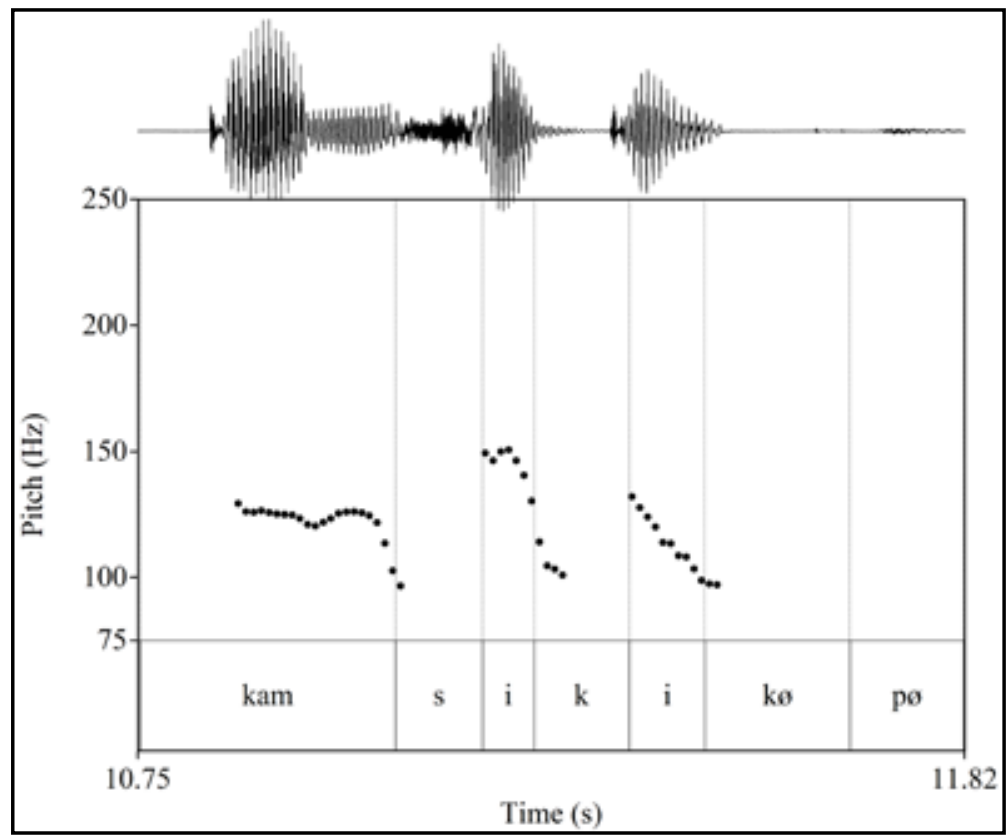

Fuente: Elaboración propia.

Otra palabra de cuatro sílabas como ['pir.ka.kÆ.na] </pirka+plural/ en la frase 'estos muros' evidencia el contorno tonal alto en la primera sílaba de la palabra con un descenso en las demás sílabas posteriores a esta, como se observa en la figura 4. En dicho caso, se reporta la elisión de la vocal alta dorsal del sufijo de plural /-kuna/ > [-kna]. 
Figura 4. Contorno tonal de palabra ['pir.ka.kØ.na.] </pirka+plural/

\section{en la frase 'estos muros'}

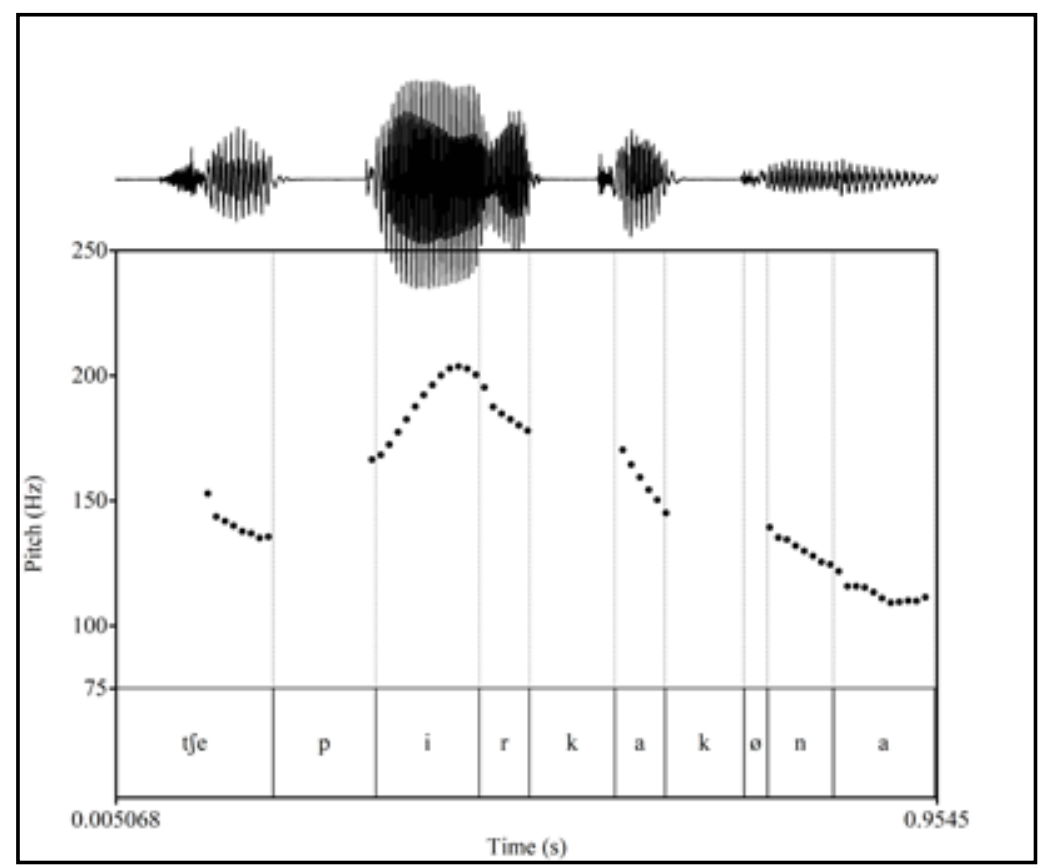

Fuente: Elaboración propia.

De la misma manera, si formamos una palabra con seis sílabas como para el caso de ['ru.na.kÆ.na.ta] </runa+plural+acusativo/ 'a los hombres', el contorno tonal alto se sigue manteniendo en la primera sílaba de la palabra con el descenso en las siguientes. Sin embargo, como se observa en la figura 5, además de lo descrito arriba, se atestigua un ascenso particular del contorno tonal en la sílaba [na] como si fuera un acento secundario. 
Figura 5. Contorno tonal de palabra ['ru.na.kØ.na.ta]

$</$ runa+plural+acusativo/

'a los hombres'

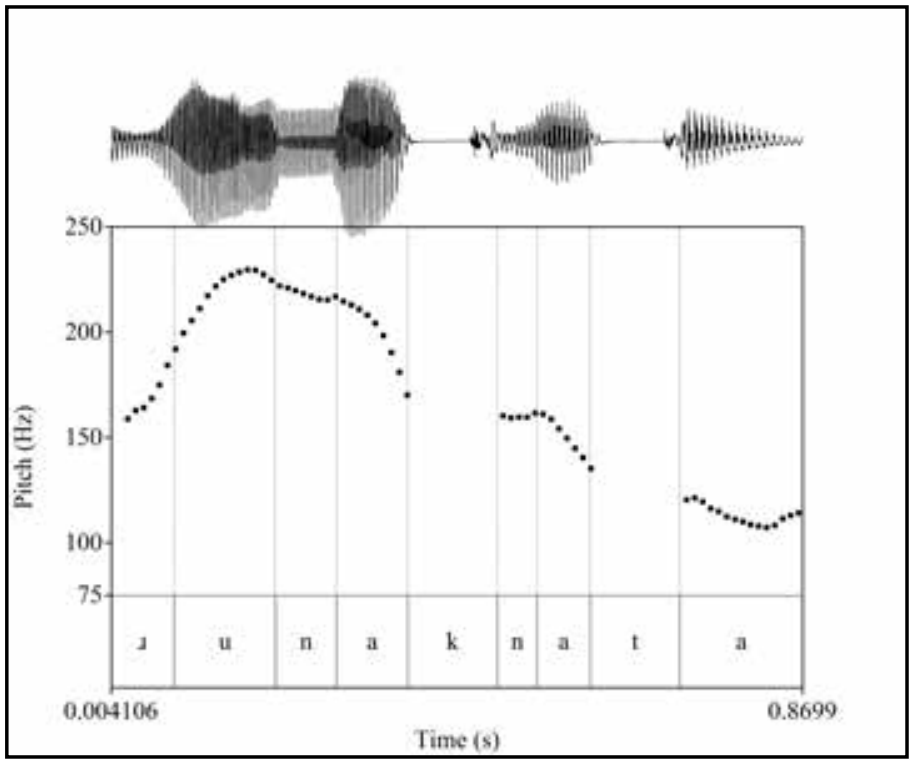

Fuente: Elaboración propia.

La presencia de este contorno tonal, que al parecer representa un acento secundario, se atestigua con mayor frecuencia en los verbos. En este caso, cuando la sílaba está constituida por una consonante en posición coda y no final de palabra, esta parece atraer un acento secundario y en algunas ocasiones hasta el acento primario. Como se muestra en la figura 6, la palabra ['Ja., mur.kay] </ Jamu $+\mathrm{rka}+\mathrm{n} /$ 'él vino', con una configuración silábica $<$.cv.cvc.cvc. $>$, presenta un contorno tonal alto tanto en la primera sílaba [ $\left.\mathrm{\int a}\right]$ como en la segunda [mur], y un contorno tonal bajo en [kan]; sin embargo, el oscilograma registra que es la primera sílaba [fa] la que posee mayor energía acústica, por lo que nuestra interpretación asume a esta con el acento primario y a la segunda con el secundario. 
Figura 6. Contorno tonal de palabra ['fa., mur.kay $]</$ Jamu+rka+n/ 'él vino'

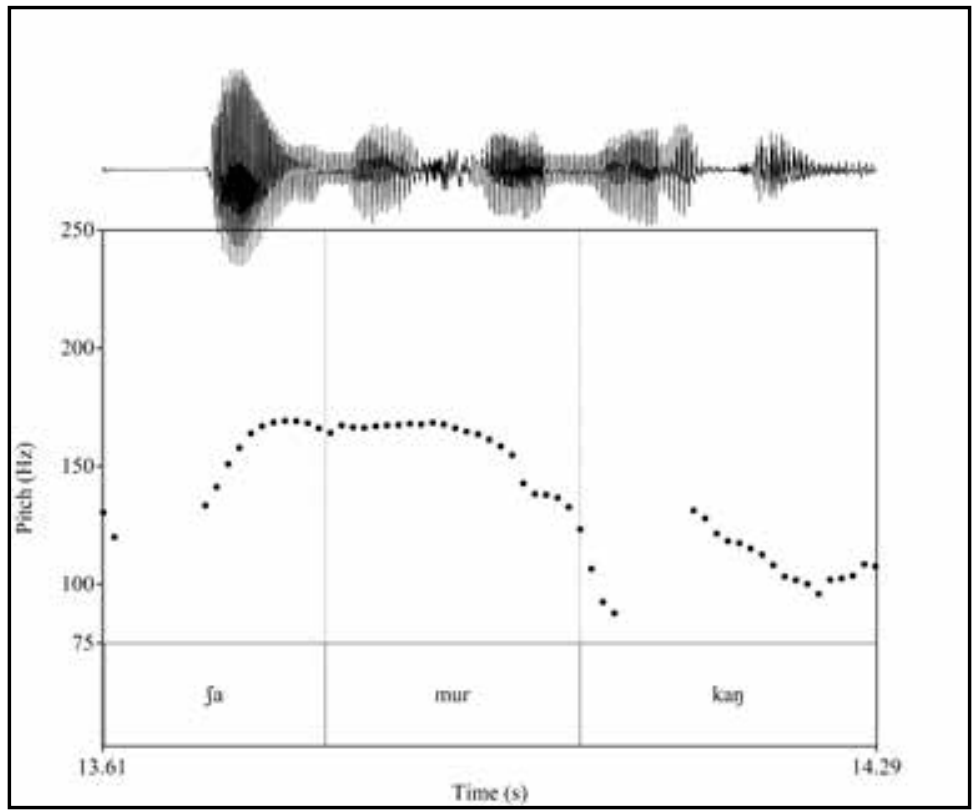

Fuente: Elaboración propia.

Si añadimos un sufijo más a / Jamu-rka-n/ 'él vino', como por ejemplo para el caso de ['Ja.mur.,kan.na] < / $a m u+r k a+n+n a /$ 'él ya vino', con una configuración silábica <.cv.cvc.cvc.cv>, observaremos que el contorno tonal alto aparece tanto en la primera como en la segunda sílaba, pero ahora la tercera sílaba muestra un comportamiento tonal distinto en relación con la última sílaba. Como se puede observar en la figura 7, el contorno tonal en la penúltima sílaba [kan] presenta un pico prominente. Esta observación nos lleva a pensar en la presencia de un acento secundario en esta sílaba en los verbos. 
Figura 7. Contorno tonal de palabra [' 'a., mur., kan.na] $</$ Jamu+rka+n+na/ 'él ya vino'

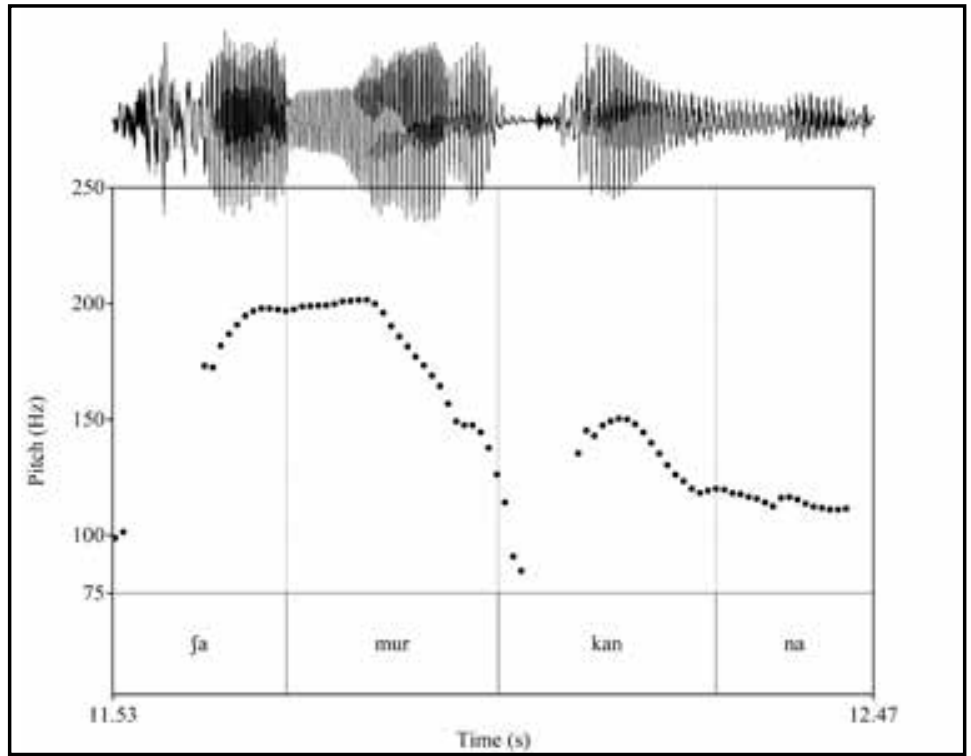

Fuente: Elaboración propia.

Este acento secundario en el verbo puede aparecer en algunos casos como acento principal, según se evidencia en la palabra [ri.ma.' Je:k.sa] </rima-Jaykisapa/ 'yo les hablo'. En esta palabra, el acento principal recae en la penúltima sílaba, como se puede observar en la figura 8. Este comportamiento acentual se distancia con lo presentado hasta ahora para el quechua chachapoyano, pero se muestra totalmente plausible con lo atestiguado para las variedades quechuas de Ferreñafe y Huancapón. 
Figura 8. Contorno tonal de palabra [ri.ma.' fe:k.sa] </rima-fayki-sapa/ 'yo les hablo'

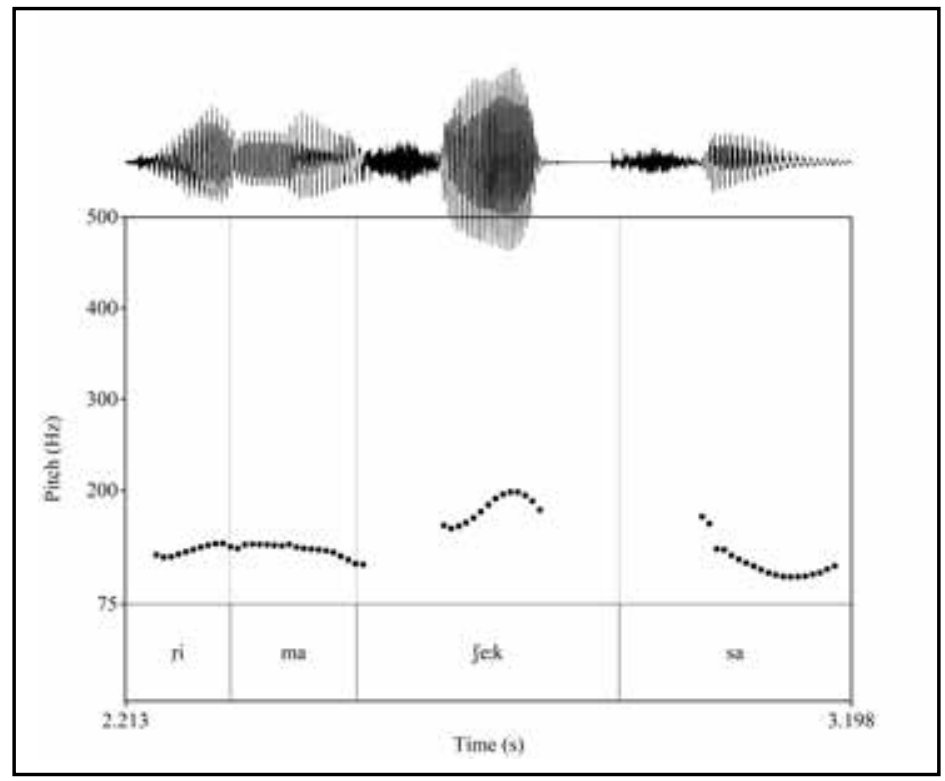

Fuente: Elaboración propia.

\section{A modo de conclusiones}

La evidencia arriba expuesta sobre el acento en el quechua de Chachapoyas demuestra un patrón acentual. Según este, el acento recae regularmente en la primera sílaba en palabras de tres hasta de seis sílabas en los nombres, observándose otro patrón en el cual el acento puede recaer en la primera sílaba o en la penúltima en los verbos. Además, se ha atestiguado la presencia de un posible acento secundario motivado rítmicamente en las palabras de cuatro sílabas en nombres o motivado por un posible peso silábico en los verbos. De manera similar, de acuerdo con los datos reportados por Pineda (1994) para el quechua de Huancapón, el acento de palabras constituidas por dos o tres sílabas en nombres recae en la primera, con la presencia de un acento secundario en las palabras trisilábicas como aparecen en (1). 
(1)

$$
\begin{array}{ll}
\text { [nó.Ga] } & \text { 'yo' } \\
\text { [kín.ray] } & \text { 'falda del cerro' } \\
\text { [á.Xa.lìn] } & \text { 'tripas' } \\
\text { [sár.si.yò:] } & \text { 'arete' }
\end{array}
$$

Si se adicionan sufijos a lexemas nominales constituidos por una o dos sílabas en la variedad de Huancapón, el acento principal se mantiene en la primera y el acento secundario puede aparecer en el último o penúltimo lugar como ocurre en (2). Estos datos muestran, además, una fluctuación del acento secundario que puede caer en la sílaba siguiente después del acento principal, como sucede cuando se añade el sufijo plural/-kuna/, que, según la autora, "mantiene su propio acento, que viene a ser secundario" (Pineda, 1994, p. 41).

$\begin{array}{ll}\text { [nó.Ga.tà] } & \text { 'yo + acusativo', } \\ \text { [páy.kù.na] } \sim \text { [páy.kùn] } & \text { 'ellos' } \\ \text { [sáy.ku.nà] [sáyknà] } & \text { 'esos' } \\ \text { [sáy.kù.na] } \sim \text { [sáy.kùn] } & \text { 'esos' } \\ \text { [Gám.kù.na] } & \text { 'vosotros, ustedes' } \\ \text { [páy.ku.ná.ta] } & \text { 'ellos + acusativo' }\end{array}$

Siguiendo con el quechua de Huancapón, Pineda (1994) registra escasas palabras de tres sílabas con acento en la penúltima, como se muestra en (3.a) y una gran cantidad de palabras cuyo acento es penúltimo motivado por el tipo de sílaba CVC, como se muestra en (3.b).

a.
[an.tfá.na]
cesto de soguilla
[wan. tfá.ko]
especie de ave 
b.
[sa.kál.wa]
quijada
[may. tfó:.pis]
en donde también
[tfaj.náX.ta]
este lugar
[wa.rán.tin]
con el día de mañana

En palabras de cuatro sílabas, al parecer el acento es regularmente penúltimo sin importar si la sílaba es CVC o CV, como se observa en (4.a). Finalmente, en palabras de cinco sílabas a más, según la autora, las "sílabas son pronunciadas con cumbre acentual cada dos sílabas” (Pineda, 1994, p. 46) como se observa en (4.b).

a.

$\begin{array}{ll}\text { [Gòngorné:wan] } & \text { con mi rodilla } \\ \text { [àlgutanó:mi] } & \text { como a perro } \\ \text { [noGànsikúna] } & \text { nosotros }\end{array}$

b.

$\begin{array}{ll}\text { [pálla.kùnatápis] } & \text { pallas }+ \text { acus. }+ \text { también } \\ \text { [mánka.kùnawánmi] } & \text { con las ollas } \\ \text { [wáyila.kùnamánmi] } & \text { hacia las casitas }\end{array}$

En Cajatambo, de acuerdo con los datos proporcionados por Carreño (2010), el acento regularmente recae en la primera sílaba tanto en nombres como en verbos. Según el citado autor, "el acento cajatambino es un acento fijo y de tonalidad, que se coloca, siempre y solamente, en la primera sílaba de la palabra" (Carreño, 2010, p. 54). Su acento tonal es peculiar y se mantiene también en el castellano andino de la zona.

Lo más curioso es su realización fonética; es un acento tonal, que consiste en una ligera elevación en el tono de la voz que acompaña al inicio de cada palabra. Esa continua sucesión de elevaciones del tono, que se da en toda frase quechua, es lo que le da esa especie de "canto" tan característico al idioma; y no solo al quechua, sino también al castellano andino, que conserva algo de la entonación quechua. (Carreño, 2010, p. 54) 


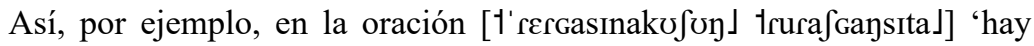
que enseñarnos nuestros trabajos', tanto la frase nominal como verbal presentan acento tonal alto en la primera sílaba de la palabra. El autor no registra un posible acento secundario en sílabas CVC, como se puede observar en (5).

$$
\begin{aligned}
& \text { Rirqa-si-na-ku-shun rura-shqa-nsi-ta. } \\
& \text { 'ver'-cau.-rec.-refl.-4 }{ }^{a} \text {.fut. 'hacer'-p.pf. } 4^{a} \text {-ac. } \\
& \text { [1'rergasinakofon」 trurafgaysita」] } \\
& \text { "Hay que enseñarnos nuestros trabajos". }
\end{aligned}
$$

Para el quechua de Ferreñafe, como se ha señalado anteriormente, Escribens (1977) atestiguó, alternancias acentuales en la primera sílaba o en la penúltima en los nombres. El primer patrón se ha atestiguado para el quechua de Chachapoyas y en palabras hasta de tres sílabas para el quechua de Huancapón. Cabe señalar, además, que las dos pautas de acentuación propuestas por Escribens se explicaban mejor mediante el establecimiento de una relación directa con las estructuras sintácticas; en estas, el núcleo de la frase verbal presenta un patrón en el cual el acento recae en la penúltima sílaba de la palabra y en el complemento donde recaía en la primera sílaba del lexema, como en [míkunata tfatfímuy] < / mikunata $t$ fat $\int i m u y /$ 'traen comida'.

Por otra parte, según los datos reportados por Torero (1964), en el habla de Huaraz (Áncash), el acento tomaba en cuenta el tipo de sílaba que compone una palabra. Si la palabra estaba compuesta solo por sílabas breves, o sea, aquellas que se estructuran con una consonante y una vocal $(\mathrm{CV})$, el acento recaía en la sílaba inicial. Si la palabra estaba compuesta por sílabas pesadas CVC o CV (largas para Torero), el acento recaía en estas. Si había varias sílabas pesadas, según el autor, el acento se producía con un relieve relativamente igual:

La sílaba larga destaca dinámica y melódicamente en la palabra, máxime si contiene vocal larga, si hay varias largas, puede destacar la última o la que posee vocal larga, o "repartirse" la intensidad articulatoria entre ellas de modo tal que se pronuncien con fuerza y altura sensiblemente iguales. Si todas las sílabas son breves, lleva el relieve la sílaba inicial $\mathrm{u}$, ocasionalmente, la final terminada en vocal larga; estas sílabas se comportan así como de intensidad media. 
Cuando una sílaba llega a destacar nítidamente, en las que la preceden o las siguen pueden aparecer relieves secundarios con ritmo binario.

(Torero, 1964, p. 461)

En la variedad quechua del sur de Conchucos, de acuerdo con los datos presentados por Hintz (2006), la tendencia es a acentuar la primera sílaba de las palabras con la presencia de un acento secundario en la penúltima sílaba. Este acento secundario puede alternar en palabras de varias sílabas como se muestra en (6).

$$
\begin{aligned}
& \text { chákrantsìkkunàtarà:chir } \\
& \text { chakra-ntsik-kuna-ta-ra:-chir } \\
& \text { FIEL-12POSS-PLUR-ACC-YET-SURARI } \\
& \text { 'our field supposedly still' } \\
& \text { (Hintz, 2006, p. 480) }
\end{aligned}
$$

En resumen, como se puede apreciar en el cuadro 2, las variedades quechuas de Chachapoyas y Ferreñafe dentro del tronco Quechua II evidencian un patrón acentual por el cual el acento recaía en la primera sílaba en los nombres compuestos por tres sílabas o más (solo en sílabas ligeras tanto para nombres como para verbos en el quechua de Chancay, según los datos de fray Domingo de Santo Tomás). De igual manera, las variedades quechuas de Huancapón y Catajambo del tronco Quechua I muestran un patrón muy similar al descrito para Chachapoyas y Ferreñafe, con una alternancia que se hacía regular para la variedad de Huancapón en palabras de más de cuatro sílabas con acento primario penúltimo y acento secundario en la primera sílaba. Por otra parte, en la variedad quechua de Áncash, el patrón acentual en la primera sílaba se evidencia en palabras constituidas por sílabas ligeras, pero con el acento principal en la penúltima sílaba cuando se encontraba una sílaba pesada (CVC o CV:). Según los datos reportados por Hintz (2006) para el quechua del sur de Conchucos, el acento principal recae en la primera sílaba de la palabra con presencia de un acento rítmico desde la penúltima sílaba hacia la izquierda. 
Cuadro 2. Descripción acentual en siete variedades quechuas

\begin{tabular}{|c|c|c|c|}
\hline Variedades & Quechua I & Quechua II & Referencia \\
\hline Ferreñafe & & $\begin{array}{l}\text { Acento en la primera sílaba en } \\
\text { nombres y acento en la penúlti- } \\
\text { ma en verbos. Al parecer con mo- } \\
\text { tivación sintáctica según el autor. }\end{array}$ & Escribens (1977) \\
\hline Chachapoyas & & $\begin{array}{l}\text { Acento principal en la primera } \\
\text { sílaba en nombres y verbos con } \\
\text { alternancias rítmicas desde la } \\
\text { penúltima sílaba. Hay casos con } \\
\text { acento principal en la penúltima } \\
\text { sílaba. }\end{array}$ & Valqui (2019) \\
\hline Chancay & & $\begin{array}{l}\text { Acento en la primera sílaba en } \\
\text { palabras hasta de tres sílabas } \\
\text { en nombres o en sílabas ligeras } \\
\text { en verbos. En los demás casos, } \\
\text { acento penúltimo en nombres y } \\
\text { según la sílaba pesada en ver- } \\
\text { bos. }\end{array}$ & Santo Tomás (1951) \\
\hline Huaraz & $\begin{array}{l}\text { Acento en la primera sílaba en } \\
\text { palabras con sílabas ligeras. } \\
\text { Cuando hay sílabas pesadas, } \\
\text { el acento recae en estas. Pre- } \\
\text { senta también acento secunda- } \\
\text { rio rítmicamente. }\end{array}$ & & Torero (1964) \\
\hline Huancapón & $\begin{array}{l}\text { Acento principal en la primera } \\
\text { sílaba en palabras hasta de } \\
\text { tres sílabas. Acento principal } \\
\text { en la penúltima sílaba en pala- } \\
\text { bras de cuatro a más sílabas. } \\
\text { El acento recae también según } \\
\text { la sílaba pesada en verbos. }\end{array}$ & & Pineda (1994) \\
\hline Cajatambo & $\begin{array}{l}\text { Acento principal solo en la pri- } \\
\text { mera sílaba tanto en nombres } \\
\text { como en verbos. }\end{array}$ & & Carreño (2010) \\
\hline $\begin{array}{l}\text { Conchucos } \\
\text { (sur) }\end{array}$ & $\begin{array}{l}\text { Acento principal en la primera } \\
\text { sílaba de la palabra con pre- } \\
\text { sencia de un acento rítmico } \\
\text { desde la penúltima sílaba hacia } \\
\text { la izquierda. Se evidencia el } \\
\text { cloque acentual en palabras } \\
\text { con sílabas impares. }\end{array}$ & & Hintz (2006) \\
\hline
\end{tabular}

Fuente: Elaboración propia. 
Estos datos, en primer lugar, parecen sostener la hipótesis de que los patrones acentuales en el quechua de Chachapoyas son en realidad vestigios de un tipo prosódico más antiguo de filiación quechua y no de filiación prequechua. En segundo lugar, los patrones acentuales de las siete variedades quechuas presentadas en el cuadro 2 apuntan hacia un modelo acentual por el cual se asignaba el acento en la primera sílaba en los nombres o verbos constituidos por sílabas ligeras, en un estadio en el que las variedades quechuas de los troncos I y II aún no se encontraban escindidas ${ }^{3}$. De esta manera, las estimaciones de Torero (1964) y de Cerrón-Palomino (2003) sobre las características prosódicas del protoquechua que se podrían encontrar en las variedades quechuas de Áncash, Cajatambo, Chancay, Ferreñafe y Chachapoyas parecen ser acertadas y necesitan de estudios más rigurosos con herramientas de análisis acústico.

\section{Notas}

1 Los datos fueron obtenidos a través de un proceso de elicitación de palabras en un contexto oracional y mediante el recojo de eventos comunicativos sobre una serie de relatos que contaron los 5 colaboradores del estudio. Ellos, de aproximadamente 65 años de edad, fueron grabados en los pueblos de Cuemal, Colcamar, en la provincia de Luya, y en los pueblos de la Jalca Grande, Granada y Olleros, en la provincia de Chachapoyas. El análisis acústico sobre la duración vocálica y el contorno tonal (pitch) en palabras del quechua de Chachapoyas ha sido realizado mediante el programa Praat desarrollado por Boersma y Weenink (2008).

2 Cuando la vocal de la segunda sílaba ha sufrido un proceso de monoptongación —por ejemplo, con los sufijos de primera y segunda persona poseedora-, estas varían en el tiempo de realización. Con la primera persona poseedora, el promedio de duración de las vocales monoptongadas es de $180 \mathrm{~ms}$, y con la segunda persona poseedora es de 80 ms. Como se ha discutido en Valqui y otros (2017), el proceso de monoptongación en esta variedad quechua mantiene un alargamiento vocálico cuando a las vocales finales de las raíces nominales se les agregan el sufijo de primera persona poseedora con el rasgo [coronal]. Así, algunos ejemplos de formas resultantes son los siguientes: ['ume:] 'mi cabeza', ['maki:] 'mi mano' y ['Jungi:] 'mi corazón'.

3 Esto último reabre interrogantes específicas en torno a cuándo llegó el quechua al territorio chachapoyano (algunos investigadores sostienen que arribó con la incursión incaica a fines del siglo Xv, cfr. Kauffmann, 2017, p. 46) y cómo se explicaría su relación con otras variedades quechuas y su historia externa (cfr. Torero, 2002). Desde luego, para responder estas preguntas se necesita de la incursión de más disciplinas, como la genética. Por ejemplo, los datos lingüísticos en términos del acento distancian al quechua de Chachapoyas con el quechua de Lamas (ambos clasificados en el Quechua II-B) y se relacionan con los datos genéticos que señalan que las muestras genéticas de Chachapoyas y Lamas no están conectadas (cfr. Barbieri y otros, 2017). 


\section{Referencias bibliográficas}

Barbieri, Ch., Sandoval J. R., Valqui J., et al. (2017). Enclaves of genetic diversity resisted Inca impacts on population history. Scientific Reports, 7, 17411. doi: 10.1038/s41598-017-17728-w.

Boersma, P., y Weenink, D. (2008). Praat. Doing Phonetics by Computer. (Versión 5.3.66).

Carreño, P. (2010). Quechua de Cajatambo: un esbozo gramatical (Tesis de Maestría con mención en Lingüística). Pontificia Universidad Católica del Perú, Lima, Perú.

Cerrón-Palomino, R. (2003) [1987]. Lingüistica quechua. Cusco: Centro de Estudios Rurales Andinos Bartolomé de Las Casas.

Elías-Ulloa, J. (2011). Una documentación acústica de la lengua shipibo-conibo (pano). (con un bosquejo fonológico). Lima: Pontificia Universidad Católica del Perú.

Escribens, A. (1977). Fonología del quechua de Ferreñafe. Lima: Universidad Nacional Mayor de San Marcos.

Hintz, D. (2006). Stress in South Conchucos Quechua: A Phonetic and Phonology Study. International Journal of American Linguistics, 72, 477-521.

Kauffmann, D. (2017). La cultura chachapoyas. Lima: Cartolan Editora y Comercializadora.

Parker, G. (2013). Trabajos de Lingüistica histórica quechua. Lima: Pontificia Universidad Católica del Perú.

Pineda, E. (1994). Aspectos de la fonología del quechua de Huancapón (Cajatambo) (tesis de Licenciatura). Universidad Nacional Mayor de San Marcos, Lima.

Santo Tomás, F. (1951) [1560]. Grammatica o arte de la lengua general de los Indios de los Reynos del Peru. Edición facsimilar, con prólogo de Raúl Porras Barrenechea. Lima: Universidad Nacional Mayor de San Marcos, Instituto de Historia. 
Taylor, G. (2000). Estudios lingüisticos sobre Chachapoyas. Lima: Universidad Nacional Mayor de San Marcos, Instituto Francés de Estudios Andinos.

Torero, A. (1964). Los dialectos quechuas. Anales Cientificos de la Universidad Agraria, 2, 446-478.

Torero, A. (1975). Lingüística e historia de la sociedad andina. En Lingüistica e indigenismo moderno de América Trabajos presentados al XXXIX Congreso Internacional de Americanistas(pp. 221-259), 5. Lima: Instituto de Estudios Peruanos.

Torero, A. (2002). Idiomas de los Andes. Lingüistica e Historia. Lima: Editorial Horizonte.

Torero, A. (2007) [1974]. El quechua y la historia social andina. Lima: Fondo Editorial del Pedagógico San Marcos.

Valqui, J. (2019). Patrones acentuales en el quechua de Chachapoyas y su implicancia para la reconstrucción del protoquechua (Tesis para optar optar por el grado académico de Doctor en Lingüística). Universidad Nacional Mayor de San Marcos. Facultad de Letras y Ciencias Humanas, Lima, Perú (en proceso de aceptación).

Valqui, J., Solís, G., Faucet, C., Espinoza, F. \& Velásquez, L. (2017). Subespecificación y monoptongación en los sufijos de primera y segunda persona poseedora en el quechua de Chachapoyas. Lengua y Sociedad, $16,88-110$.

Valqui, J. \& Ziemendorff, M. (2016). Vestigios de una lengua originaria en el territorio de la cultura chachapoya. Letras, 87(125), 5-32. 